\title{
Zu den besten Arbeitgebern gehören
}

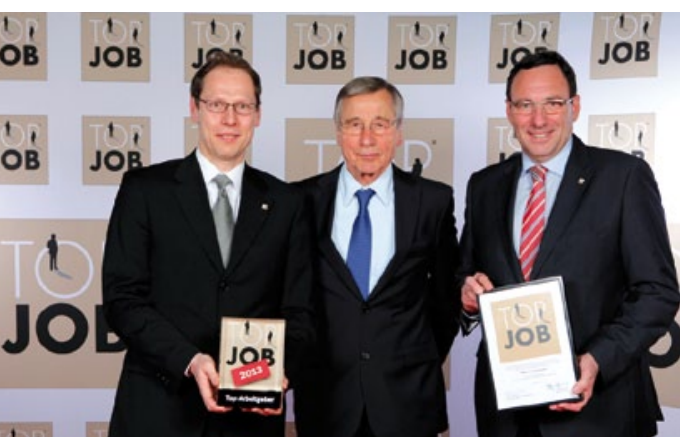

Wo finden Mitarbeiter die besten Arbeitsbedingungen im deutschen Mittelstand? Die Antwort liefert seit 2002 der von compamedia $\mathrm{GmbH}$ organisierte $\mathrm{Ar}$ beitgeber-Wettbewerb „Top Job“. Die CAMLOG Vertriebs $\mathrm{GmbH}$ gehört in diesem Jahr zum ersten Mal zu den ausgezeichneten Unternehmen. Das Institut für Führung und Personalmanagement der Universität St. Gallen befragte zuvor ausführlich die Mitarbeiter und die Personalleitungen aller Bewerber. Als Mentor des Wettbewerbs überreichte Wolfgang Clement allen Gewinnern am 31. Januar 2013 in Duisburg den Top Job-Award.

Die Qualifizierung der über 100 Beschäftigten genießt beim ausgezeichneten Unternehmen höchste Priorität. Eine gute Kommunikation und ein reibungsloser Informationsfluss sind bedeutsame Bestandteile der Firmenkultur. Bei abteilungsübergreifenden Projekten werden
Top Job-Award für die CAMLOG Vertriebs GmbH. Von links: Markus Stammen (Leiter Organisationsentwicklung), Top Job-Mentor Wolfgang Clement und Michael Ludwig (Geschäftsführer).

Ressourcen und Kompetenzen aus allen Unternehmensbereichen zusammengeführt. „Wir legen Wert darauf, dass jeder seine Anforderungen einbringt und wir das Unternehmen gemeinsam mit unseren Mitarbeitern kontinuierlich weiterentwickeln“, erläutert der Leiter der Organisationsentwicklung, Markus Stammen.

Nicht zuletzt spielen Transparenz und Vertrauen eine wichtige Rolle beim Unternehmen: „Welche Kunden die Vertriebsmanager in ihren Gebieten besuchen und mit welchen Veranstaltungen sie ihre Tätigkeit unterstützen, entscheiden sie selbst“, sagt Geschäftsführer Michael Ludwig. Sascha Wethlow, Manager Technischer Service, ergänzt: „Engagement und Eigenverantwortung sind in unserer Mitarbeiterkultur fest verankert. Der gelebte Teamgeist und die offene Kommunikation motivieren immer wieder neu. Es macht sehr viel Freude, Teil dieses innovativen und erfolgreichen Unternehmens zu sein und die Zukunft aktiv mitgestalten zu können.“

„Der Top Job-Award ist Auszeichnung und Ansporn zugleich, sowohl für mich als auch für meine Mitarbeiter“, bekräftigt Michael Ludwig und erläutert: „Wir haben gemeinsam schon sehr viel erreicht. Der Award und die Erkenntnisse aus der ausführlichen Analyse unserer Personalarbeit sind für uns zusätzliche Motivation, den erfolgreichen Weg fortzusetzen.“

Nach einer Pressemitteilung der

CAMLOG Vertriebs GmbH, Wimsheim

www.camlog.de

\section{Goldenes Doktordiplom}

\section{Vor 50 Jahren promoviert?}

Die Charité ehrt seit vielen Jahren ihre Alumni, die vor 50 Jahren an der Charité promoviert haben, mit der Vergabe einer „Goldenen Doktorurkunde“. Auch in diesem Jahr soll es wieder im Rahmen eines großen Festaktes im Konzerthaus am Gendarmenmarkt in Berlin-Mitte erfolgen. Leider ist der Kontakt zu so manchem Kollegen verloren gegangen. Sollten Sie vor etwa 50 Jahren in Berlin promoviert haben oder jemanden kennen, für den das zutrifft, melden Sie sich doch bitte im Promotionsbüro der Charité - Universitätsmedizin Berlin 030/450576018/016.

Nach einer Pressemitteilung der

Charité

Promotionsbüro

Manuela Hirche

Augustenburger Platz 1

13353 Berlin

\section{PEERS-Förderpreise erneut ausgeschrieben}

Bereits zum 5. Mal sind junge Wissenschaftler und Zahnärzte an Kliniken und in niedergelassenen Praxen aufgerufen, sich mit ihren Arbeiten zu aktuellen Themen der Implantologie um einen der PEERSFörderpreise $\mathrm{zu}$ bewerben. In 5 verschiedenen Kategorien werden die Preise, die mit 3000-6000 Euro dotiert sind, für Arbeiten mit klinischer Relevanz vergeben: Dissertation - Fallpräsentation (Poster) wissenschaftliches Poster und wissenschaftliche Publikation sowie der Implantat-Prothetik-Preis, der in Zusammenarbeit mit der Klaus-Kanter-Stiftung ausgeschrieben und verliehen wird. Die Arbeiten dürfen nicht älter als 2 Jahre sein, die wissenschaftlichen Publikationen müssen in einem Journal mit Peer-Review-Verfahren veröffentlicht worden sein.

Die PEERS-Förderpreise wurden ins Leben gerufen, um jüngere Wissenschaftler und wissenschaftlich interessierte Zahnärzte in ihrer Arbeit zu unterstützen. Initiator der Preise ist PEERS (Platform for the Exchange of Experience, Education, Research and Science), ein von DENTSPLY Implants unterstütztes, internationales Expertennetzwerk mit Mitgliedern aus Klinik und Praxis und aus dem Bereich der Zahntechnik. Alle Preisträger werden am Abend des 12. Oktober im Rahmen des 1. DENTSPLY Implants Kongresses

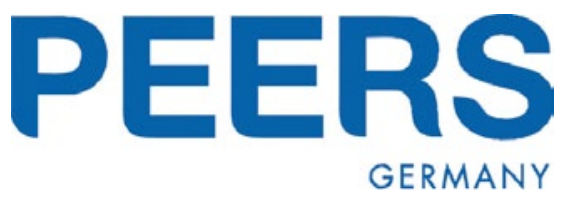

(DIKON) in Deutschland bei einer feierlichen Preisverleihung geehrt. Weitere Informationen $\mathrm{zu}$ den PEERS-Förderpreisen, Teilnahmebedingungen, eine Postervorlage und das Anmeldeformular können unter peers.foerderpreise@dentsply. com angefordert oder unter www. dentsplyimplants.de abgerufen werden. Die Arbeiten müssen bis zum 31. Juli 2013 eingereicht werden.

Nach einer Pressemitteilung der DENTSPLY Implants, Mannheim 


\section{Jetzt mit „Forum Zahntechnikerinnen“}

Die Anregung kam aus dem Kreis der Zahntechnikerinnen: Gesucht wurde eine Möglichkeit, sich ähnlich wie die Zahnärztinnen in einem eigenen Forum zu organisieren. Insbesondere die Inhaberinnen von Dentallaboren wünschten sich eine Plattform für die Kommunikation und den Erfahrungsaustausch untereinander. Hierzu gab es seit rund 2 Jahren viele auch persönliche Kontakte zwischen Zahntechnikerinnen und dem Dentista Club. Ein eigenes Projekt zu starten und neben der Leitung des Labors mit Leben zu füllen, erwies sich allerdings als zu anspruchvoll, sodass die Überlegung aufkam, sich sinnvollerweise unter dem
Dach des Dentista Clubs zu organisieren und die bestehenden Strukturen mit zu nutzen. Die Mitgliederversammlung des Dentista Clubs stimmte dem Konzept im vergangenen Sommer zu. Zum Jahresbeginn 2013 ist nun das „Forum Zahntechnikerinnen“ im Dentista Club gestartet und findet sowohl bei Zahnärztinnen als auch bei Zahntechnikerinnen deutlich positiven Widerhall.

Gründungs-Vorsitzende des Forums Zahntechnikerinnen und in dieser Funktion Mitglied des Vorstands des Dentista Clubs ist Zahntechnikmeisterin Annette von Hajmasy/Köln. „Dass der Dentista Club den engagierten Expertinnen aus

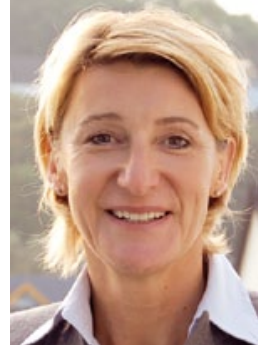

Zahntechnikmeisterin Annette von Hajmasy / Köln leitet als Gründungs-Vorsitzende das neue „Forum Zahntechnikerinnen“ unter dem Dach des Dentista Clubs.

dem Bereich Zahntechnik die Möglichkeit gibt, sich in dessen Struktur zu organisieren, ist großartig“, so ZTM von Hajmasy, „und es ist eine moderne zeitgemäße Entwicklung! “

Nach einer Pressemitteilung der Buena Vista Dentista Club e. V. Verband der Zahnärztinnen, Berlin

\section{Der schnelle Weg zum Ziel}

Schneller und besser war nicht nur ein Thema der diesjährigen IDS. Gleich ein Wochenende später nach Messe-Ende gab die 28. Karlsruher Konferenz Antwort auf dieses gern gegebene Versprechen der Industrie. Bringen die neuen Techniken und Behandlungskonzepte wirklich Zeitgewinn bei gleicher Effizienz gegenüber bewährten Verfahren? Um den Kollegen bei der Bewertung dieser Versprechen zu unterstützen, gaben 4 Referenten ihre Einschätzung auf den Gebieten Implantologie, Parodontologie, Kieferorthopädie und Endodontologie nach Auswertung umfangreicher Review-Studien.

Den Einstieg in die Implantologie gab eine Life-OP aus der Akademie. Dr. Michael Korsch, Karlsruhe, demonstrierte an 2 Sofortimplantationen 13, 16 mit Sinus-Lift und provisorischer, festsitzender Sofortversorgung, wann eine „schnelle“ Lösung möglich ist. „Ein DVT-gestütztes Vorgehen sei in jedem Fall notwendig“, erklärte Korsch bei der Demonstration und auch später bei der Diskussion. Vorangegangene apikale Prozesse sowie eine bei der Extraktion nicht zu erhaltene oder nicht vorhandene vestibuläre Knochenlamelle sind bei der Sofortimplantation kritisch zu bewerten, ebenso Weichgewebsdefizite und eine ungünstige Achsenneigung. Konsequenz: Sofort- vs. verzögerter/Spätimplantation. Bei Abwägung aller patienten- und technikbeding-

\section{Dental Academy for Continuing Professional Development}
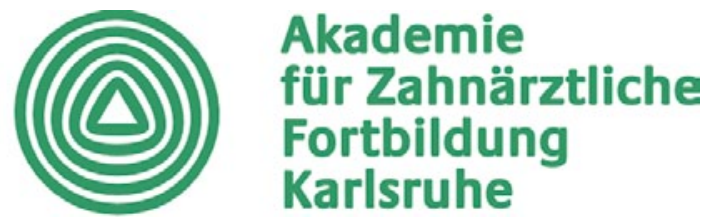

ten Risiken ist die Sofortimplantation als „schnelle“ Lösung durchaus indiziert.

Für die Parodontologie übernahm Prof. Dr. Christian Dörfer, Kiel, die Bewertung. Zur Diskussion standen Full-Mouth-Disinfektion, systematische Antibiotikatherapie, Photodynamik, Probiotika sowie die primär offene Curettage. Alle Therapieansätze seien erfolgreich, eine echte Zeitersparnis bei gleichzeitiger Effizienz bieten sie aber nicht. Besonders zu beachten sei, dass die systemische Antibiose nur in ausgewählten Fälle indiziert und die lokale eher als „letzte Maßnahme“ zu bewerten ist. Konsequenz: neue Konzepte vs. konventionelle Therapie. Der schnellste Weg ist eine stringent geplante, klar strukturierte und gut durchgeführte konventionelle Therapie bei lebenslanger Nachsorge.

Prof. Dr. Edgar Schäfer, Münster, konnte für den Endo-Bereich in der Tat Positives berichten. Zeitersparnispotenzial sei möglich bei der Kanalaufbereitung und der -füllung. Die neuen Ein- und auch Zweifeilensysteme zeigen sehr gute Ergebnisse bei der Aufbereitung. Die Feilengeometrie vergrößert den Spanraum, so- dass mehr Debris in kürzerer Zeit entfernt werden kann. Die Verkürzung der Aufbereitung geht aber auf Kosten der Spülzeit mit NaOCl. Eine Spülung mit Ultraschallaktiviertem $\mathrm{NaOCl}$ (unter 6\%) kann das ausgleichen. Durch die Bewegung des Ansatzes und die damit verbundene Erwärmung der Flüssigkeit ist eine 6- bis 7-Fache höhere Bakterienfreiheit zu erzielen. Die zu allen Feilensystemen passenden Guttapercha-Stifte und ein guter Sealer ermöglichen abschließend eine sichere Wurzelkanalfüllung. Konsequenz: Mehrfeilen- vs. Einfeilensystem. Eine erhebliche Zeitersparnis bei gewünschter Effizienz mit den Einfeilensystemen, mit Ultraschall-aktivierter $\mathrm{NaOCl}$-Spülung und einem Sealer auf Epoxidharzbasis.

Das Thema der Konferenz fand ich sehr mutig, stellte sie doch einige Techniken und Konzepte auf den Prüfstand. Fazit für mich: Schneller und besser klingt sehr verführerisch, hält aber oft nicht, was es verspricht. Besser und schneller wäre die richtige Reihenfolge. Dazu können neue Behandlungskonzepte und -techniken durchaus beitragen, vorausgesetzt man beherrscht sie. 